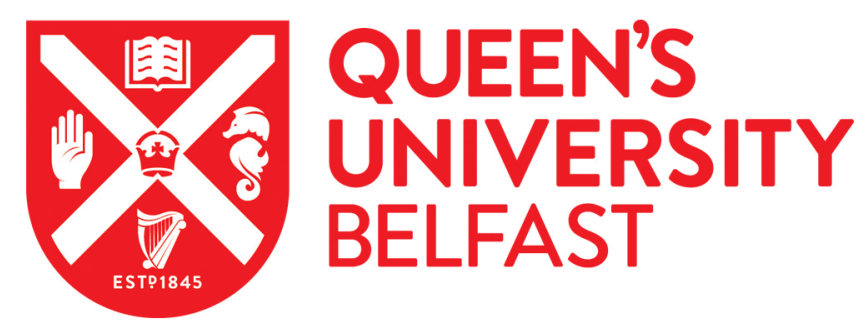

\title{
Comparison of Hybrid RANS-LES Simulations of Turbulent Flow and Heat Transfer in a Ribbed Duct
}

Kim, S., \& O'Sullivan, A. (2018). Comparison of Hybrid RANS-LES Simulations of Turbulent Flow and Heat Transfer in a Ribbed Duct. In Progress in Hybrid RANS-LES Modelling: Papers Contributed to the 6th Symposium on Hybrid RANS-LES Methods, 26-28 September 2016, Strasbourg, France (Vol. 137, pp. 513524). Springer. https://doi.org/10.1007/978-3-319-70031-1_43

Published in:

Progress in Hybrid RANS-LES Modelling

Document Version:

Peer reviewed version

Queen's University Belfast - Research Portal:

Link to publication record in Queen's University Belfast Research Portal

Publisher rights

(c) Springer International Publishing AG 2018. This work is made available online in accordance with the publisher's policies. Please refer to any applicable terms of use of the publisher.

\section{General rights}

Copyright for the publications made accessible via the Queen's University Belfast Research Portal is retained by the author(s) and / or other copyright owners and it is a condition of accessing these publications that users recognise and abide by the legal requirements associated with these rights.

Take down policy

The Research Portal is Queen's institutional repository that provides access to Queen's research output. Every effort has been made to ensure that content in the Research Portal does not infringe any person's rights, or applicable UK laws. If you discover content in the Research Portal that you believe breaches copyright or violates any law, please contact openaccess@qub.ac.uk. 


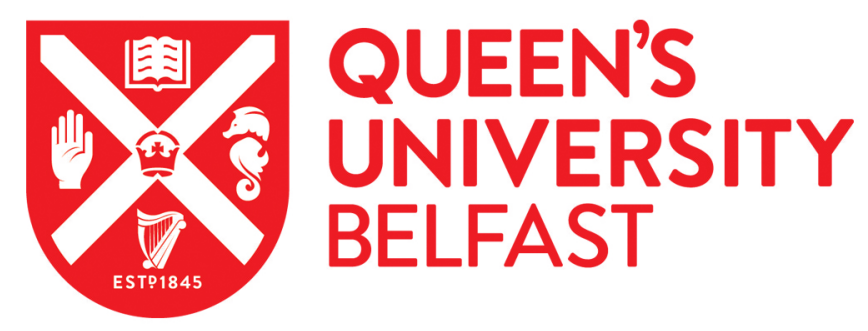

\section{Comparison of Hybrid RANS-LES Simulations of Turbulent Flow and Heat Transfer in a Ribbed Duct}

Kim, S., \& O'Sullivan, A. (2018). Comparison of Hybrid RANS-LES Simulations of Turbulent Flow and Heat Transfer in a Ribbed Duct. In Progress in Hybrid RANS-LES Modelling: Papers Contributed to the 6th Symposium on Hybrid RANS-LES Methods, 26-28 September 2016, Strasbourg, France (Vol. 137, pp. 513524). Springer. DOI: 10.1007/978-3-319-70031-1_43

Published in:

Progress in Hybrid RANS-LES Modelling

Document Version:

Peer reviewed version

Queen's University Belfast - Research Portal:

Link to publication record in Queen's University Belfast Research Portal

\footnotetext{
General rights

Copyright for the publications made accessible via the Queen's University Belfast Research Portal is retained by the author(s) and / or other copyright owners and it is a condition of accessing these publications that users recognise and abide by the legal requirements associated with these rights.
}

Take down policy

The Research Portal is Queen's institutional repository that provides access to Queen's research output. Every effort has been made to ensure that content in the Research Portal does not infringe any person's rights, or applicable UK laws. If you discover content in the Research Portal that you believe breaches copyright or violates any law, please contact openaccess@qub.ac.uk. 


\title{
Comparison of hybrid RANS-LES simulations of turbulent flow and heat transfer in a ribbed duct
}

\author{
Sung in Kim* and Aidan O'Sullivan \\ Queen's University Belfast, UK
}

\begin{abstract}
Numerical predictions of the turbulent flow and heat transfer of a stationary duct with square ribs $45^{\circ}$ angled to the main flow direction are presented. The rib height to channel hydraulic diameter is 0.1 , the rib pitch to rib height is 10 . The calculations have been carried out for a bulk Reynolds number of 50,000. The flows generated by ribs are dominated by separating and reattaching shear layers with vortex shedding and secondary flows in the cross-section. The one of hybrid RANS-LES approaches, Detached Eddy Simulation (DES), is adopted to simulate such flows at a reasonable computation cost. The capability of the various versions of DES method, depending on the RANS model, such as SpalartAllmaras model (SA), Realizable k- $\varepsilon$ model (RKE) and Shear Stress Transport (SST) model, has been compared and evaluated against the experimental data. The significant effect of RANS model on the accuracy of the DES prediction has been shown. The DES-SST method, which was able to reproduce the reasonable physics of flow and heat transfer in a ribbed duct, showed better performance than others.
\end{abstract}

\section{INTRODUCTION}

Gas turbine engines are designed to be run at very high temperatures in order to increase the thermodynamic efficiency of the engine. The melting points of the materials of turbines create a constraint on the temperature that the engine can operate at. To facilitate higher engine temperatures various cooling techniques have been incorporated into the design of the gas turbine engines. The cooling of the turbine blades has allowed the engines to be run at temperatures exceeding the melting points of the turbine blade materials. To enhance the heat transfer in the cooling passages of the blade, turbulators are installed in the cooling duct. One of the extensively used turbulators is the form of ribs placed incrementally along the walls of the duct. These ribs increase the near wall turbulence by tripping the boundary layer, which increases heat transfer rate between the coolant flow and the blade, but only has a relatively small pressure drop.

Turbine blades designers need to understand the flow physics in the ribbed cooling passages of the blade to optimise the performance of the cooling channels. A greater understanding is being achieved through the experimentation with physical test rigs and CFD simulations. In order to simulate this kind of turbulent flows as accurately as possible an extensive understanding of both the flow features pre-

\footnotetext{
* Corresponding author: s.kim@qub.ac.uk
} 
sent and the general suitability of various turbulence models for the problem is necessary. Large, geometry dependent, coherent vortices are dominant in this flow region. The Reynolds-averaged Navier-Stokes (RANS) approach generally performs poorly to simulate such features. Ribbed channel flows have been investigated using various RANS turbulence models. As a result, RANS model performances tend to be problem dependent. Also, better modelling near-wall flow behaviour is required to predict the heat transfer performance accurately.

Eddy resolving schemes, such as Large Eddy Simulation (LES), are accurately predicting large complex turbulent flows in various cases. Sewall et al. [1] compared LES with previous experimental data and found that it predicted the heat transfer and flow characteristics accurately in both the developing and developed flow regions of a stationary ribbed duct. Their LES results predicted the local and global heat transfer results within experimental uncertainty. However, the required computing resources were significant; 20 million cells, 344 processors, and about $0.5 \mu \mathrm{s} /$ grid node of wall clock time. LES models would frequently be too computationally expensive for practical applications, often being between 10 to 100 times more expensive than RANS [2]. This has resulted in the development of hybrid RANS-LES models, such as Detached Eddy Simulation (DES). In some cases, these can achieve similar levels of accuracy as LES approach, while having much less computational expense due to regions resolved by RANS.

DES is the most popular model used to date, simply consisting of changing the RANS behaviour to LES behaviour based on the either the distance to the nearest wall or the turbulence length scale. Viswanathan and Tafti [3] investigated the accuracy of SST based DES for stationary and rotating ducts by comparing with the results of LES and RANS simulations. They found that the DES model achieved similar levels of accuracy as the LES model, but the computational cost is approximately a tenth of the LES model for the conditions present. It provided more accurate results with respect to secondary flows than those of RANS and URANS, however, produced much thicker shear layer than it should be.

The main objective of this study is to compare the accuracy and suitability of various DES models, particularly, depending on the different RANS models, for the prediction of both the flow features and heat transfer characteristics in the flow field of an internal ribbed duct. The results obtained for both heat transfer and fluid flow are analysed and compared to available experimental results.

\section{Test Case: a flow in a ribbed duct}

The case analysed is a straight ribbed square duct section. As shown in Fig. 1, the bottom half of a two wall ribbed duct is modelled and thus a symmetry boundary condition is used at the top surface of the computational domain. The domain tested includes six $45^{\circ}$ angled ribs which result in strong secondary flows and increase heat transfer. In the present study, the aspect ratio of the stationary channel is one, and the rib pitch to rib height $(\mathrm{P} / \mathrm{e})$ ratio is 10 . The hydraulic diameter $\left(\mathrm{D}_{\mathrm{h}}\right)$ of the channel is $100 \mathrm{~mm}$. The rib height to hydraulic diameter $\left(\mathrm{e} / \mathrm{D}_{\mathrm{h}}\right)$ is 0.1 . The Reynolds number of 50,000 was selected based on the range of real gas turbine 
cases, as well as to compare with the experimental test case [4]. The test section involves the inlet section of $600 \mathrm{~mm}$, which allows the flow to fully develop before the ribbed section. Six ribs are then placed to allow the flow to approach a fully developed case. The outlet section after the ribbed section is two and half pitches long in order to prevent any unwanted effects from the outlet.

The simulation results are compared to the experimental results of Chanteloup et al. [4] where the practical geometries and the results of $45^{\circ}$ angled ribbed duct were provided. Therefore, the configuration of the test case is based on the experiments [4]. Their experimental uncertainty was estimated as $8 \%$ for both the heat transfer and flow measurements.

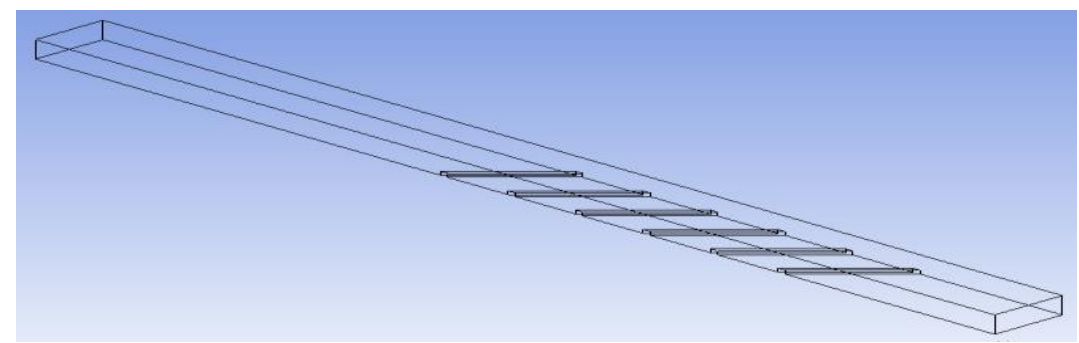

Figure 1. Computational domain, the half of a two wall ribbed duct

\section{Computational approach}

All the simulations are carried out using a commercial CFD package, ANSYS FLUENT R15. The fluid domain was defined by four boundary conditions: velocity inlet, pressure outlet, no-slip wall and symmetry boundary conditions. At the inlet, the velocity of the air was specified at $7.82 \mathrm{~m} / \mathrm{s}$ in order to give a Reynolds number of 50,000 (corresponding to the flow conditions of the experiment [4]). In order to analyse the thermal performance by scrutinizing the nondimensional heat transfer coefficient, Nusselt number, the wall heat flux to heat the main flow was specified as $300 \mathrm{~W} / \mathrm{m}^{2}$. A symmetry condition was specified at the top surface of the computational domain, corresponding to the mid-plane of the square duct (the computational domain in Fig. 1 is only the half of the square duct).

Detached Eddy Simulation (DES) is a three-dimensional unsteady numerical solution using a single turbulence model. DES involves the RANS treatment of attached turbulent boundary layers, whilst LES is applied to regions of massively separated flow (only detached eddies are simulated). DES method should be capable of modelling an entire boundary layer with RANS. A single turbulence model functions as a subgrid-scale model in the regions where the grid density is fine enough for Large-Eddy Simulation.

In ANSYS FLUENT, three RANS models are available for DES method, namely, Spalart-Allmaras model (SA), Realizable k- $\varepsilon$ model (RKE) and Shear Stress Transport (SST) model. The manner in which the DES model switches between RANS and LES modes is depending on the RANS equation which DES is based on. The first approach is based on the Spalart-Allmaras (SA) turbulence 
model. This method uses the distance from the wall, $d$, to determine the local length scale. The length scale for this DES-SA model is then subsequently defined as,

$$
\tilde{d}=\min \left(d, C_{D E S} \Delta_{\max }\right)
$$

where the grid spacing, $\Delta_{\max }$, is based on the largest mesh size in the $\mathrm{x}, \mathrm{y}$, or $\mathrm{z}$ directions. The empirical constant $\mathrm{C}_{D E S}$ is 0.65 .

This should ensure that the RANS model is used throughout all of the entire boundary layer, as for most cases, $\Delta_{\max }$ will exceed the boundary layer thickness, $\delta$. For a typical RANS grid with a high aspect ratio in the boundary layer, the wall-parallel grid spacing usually exceeds $\delta$. However, if $\Delta_{\max }$ is smaller than $\delta$, then DES can activate the LES mode inside the boundary layer. In such a case, the Delayed Detached Eddy simulation (DDES) model, which is available in ANSYS Fluent, can be used.

The corresponding modification to the DES length scale are

$$
\tilde{d}=d-f_{d} \max \left(0, d-C_{D E S} \Delta_{\max }\right)
$$

Where the shield function, $f_{d}$ is given by

$$
f_{d}=1-\tanh \left(\left(8 r_{d}\right)^{3}\right) \quad \text { and } \quad r_{d}=\frac{\tilde{v}}{\sqrt{U_{i, j} U_{i, j}} \kappa^{2} d^{2}} ; \kappa=0.41
$$

The boundary layer sensor function, $r_{\mathrm{d}}$, is equal to one in the logarithmic layer and vanishes at the edge of the boundary layer. The hyperbolic tangent blending function, $f_{\mathrm{d}}$, was tuned such that the earliest onset of LES mode occurs just outside the boundary layer. The function assumes the value $f_{\mathrm{d}}=0$ inside the turbulent boundary layer, blending smoothly to $f_{\mathrm{d}}=1$ at the boundary layer edge. In the present simulations, the delayed DES was used as a default setting.

In the realizable $k-\varepsilon$ model based DES (DES-RKE), the RANS model is almost same to the realizable $k-\varepsilon$ model with only the modification of the dissipation term in the $k$ equation. In the DES-RKE model, the RANS dissipation term is modified

such that: $\quad Y_{k}=\frac{\rho k^{3 / 2}}{l_{D E S}}$

and $\quad l_{D E S}=\min \left(l_{R K E}, l_{L E S}\right), l_{R K E}=\frac{k^{3 / 2}}{\varepsilon}, l_{L E S}=C_{D E S} \Delta_{\max }$

where $C_{D E S}$ is a calibration constant with a value of 0.61 and $\Delta_{\max }$ is the maximum local grid spacing in the $\mathrm{x}, \mathrm{y}$, or $\mathrm{z}$ directions. Similarly, the delayed concept has been applied to the DES-RKE model to preserve the RANS mode throughout the boundary layer. In two equation model based DES, $\tilde{v}$ of the function, $r_{\mathrm{d}}$, for DES-SA is replaced by $v_{t}+v$. The DES length scale is redefined as

$$
l_{D E S}=l_{R K E}-f_{d} \max \left(0, l_{R K E}-l_{L E S}\right)
$$

The last method is the SST model based DES (DES-SST). Unlike the DES-SA approach, the DES-SST method takes account of the local flow conditions. In the DES-SST model, the RANS dissipation term is modified such that: 


$$
Y_{k}=\rho \beta^{*} k \omega F_{D E S} \text { and } \quad F_{D E S}=\max \left(\frac{l_{k-\omega}}{l_{L E S}}\left(1-F_{S S T}\right), 1\right), l_{k-\omega}=\frac{k^{1 / 2}}{\beta^{*} \omega}
$$

where $F_{S S T}=0, F_{1}, F_{2}$ are the blending function of SST and $l_{k-\omega}$ is the RANS length scale. $\mathrm{C}_{\mathrm{DES}}$ is equal to 0.61 , when the $\mathrm{k}-\omega$ model is operating and equal to 0.78 for the $\mathrm{k}-\varepsilon$ model. Therefore, when the length scale derived from the grid is larger than the eddy length scale, the RANS model is activated. This ensures the model operates in RANS mode in the boundary layer, but LES is also operates in other regions where turbulent length scale is larger than the grid.

In the present simulation, convective fluxes are computed with a second-order central-differencing scheme. The time-marching scheme is based on a secondorder implicit approach. The unsteady simulation commenced from the initial condition, which is the converged solution of the steady SST simulation. The characteristic time of the internal flow, the hydrodynamic diameter, $\mathrm{D}_{\mathrm{h}}$, divided by the mean velocity, is around $0.013 \mathrm{~s}$ in the present case. The through-flow time, the time for a particle dropped at the inlet of the domain to reach the outlet, is around $0.187 \mathrm{~s}$ in the present domain. A time step size of $\Delta \mathrm{t}=0.1 \mathrm{~ms}$ is used in all of the cases. The initial transient period of 4000 time steps is ignored prior to obtaining unsteady statistical data. It corresponds to the period of longer than two through-flow times. The mean characteristics of the flow and heat transfer are obtained by averaging the solution for an additional 4000 time steps.

To achieve a mesh which captures the flow features present, accurately, a grid independence study was carried out using steady RANS simulation with SST model. Each inter-rib section in the computational domain has the same element distribution. The elements were clustered towards each solid surface to capture the regions of high turbulent shear layer and boundary layer. It is also important to ensure that the massive separation and large eddies around the rib were well captured. These are crucial to the accurate prediction of flow features as well as heat transfer. The average $\mathrm{y}^{+}$values of all the meshes were kept lower than 1 . Even though there is a significant bunching of elements towards each of walls, the ratio set for the element growth rate was never greater than 1.1. Each of the periodic ribbed sections in the coarse mesh has 60 elements placed on the horizontal, verti$\mathrm{cal}$ and in plane edges. For the medium and fine meshes, the coarse mesh was refined by a factor of 1.2 and 1.44 , respectively. The total numbers of elements for coarse, medium and fine meshes are 2.2 million, 3.7 million and 6.6 million. The discrepancy of reattachment distance, i.e. the distance between the trailing edge of the rib and the point where the boundary layer begins to develop, between coarse and medium, as well as medium and fine are around $4 \%$ and $1 \%$, respectively. The key velocity profiles of three meshes have a significant level of overlap. In order to sufficiently resolve turbulence eddies, the fine mesh has been used in the present study. 


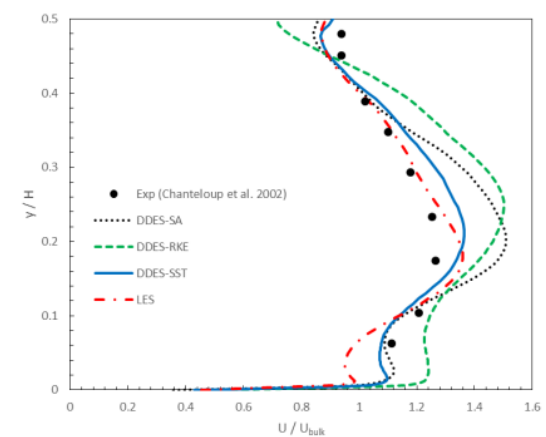

(a)

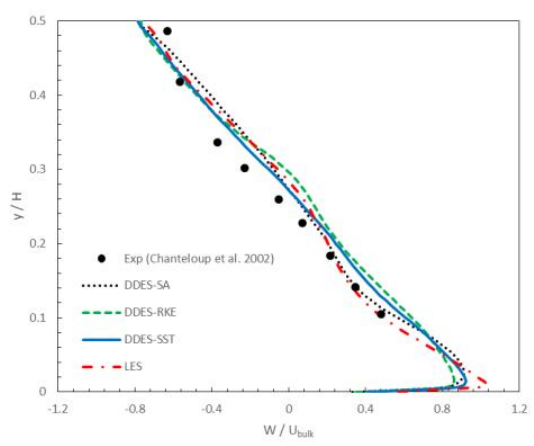

(b)

Figure 2. Comparison of the averaged velocity profiles in the center plane of the duct and at the mid point between $5^{\text {th }}$ and $6^{\text {th }}$ rib, (a) u-velocity and (b) wvelocity

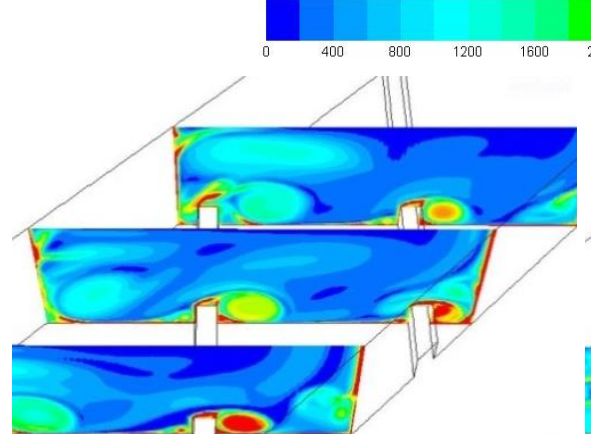

(a) Steady - SST

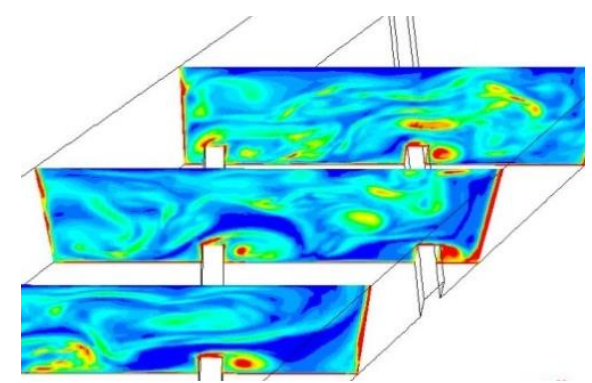

(c) DDES - RKE

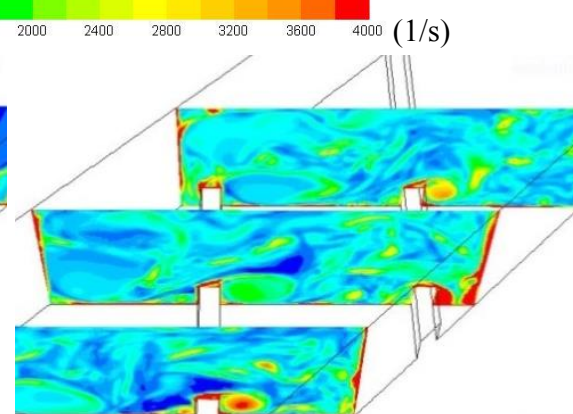

(b) DDES - SA

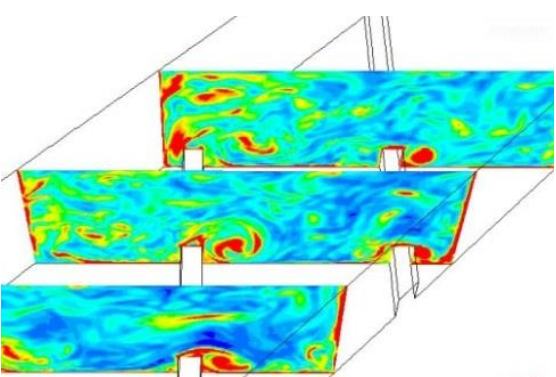

(d) DDES - SST

Figure 3. Contours of coherent vorticity in the planes normal to the rib 


\section{Results and discussion}

\section{$\underline{\text { Flow characteristics }}$}

Firstly, the mean velocity profiles of the DES solutions are compared to the experimental measurement for the quantitative analysis. The time averaged velocity profiles in the central plane of the duct $(\mathrm{z} / \mathrm{W}=0.5)$ and at the middle point between the fitth and sixth ribs are plotted against the experiments [4] in Fig. 2. Also, the LES results using the same mesh are shown. Except the near wall region, the averaged $u$ velocity of LES shows the best agreement with the experimental measurement. The worst prediction is obtained by DDES-RKE since it over-predicts the overall $\mathrm{u}$ velocity profile. The DDES-SST is the best among the DES methods as well as comparable to the LES results. Furthermore, DDESSST shows better near wall prediction than that of LES. Therefore, it is confirmed that the drawback of LES is near wall treatment and the appropriate choice of RANS model in DES method can provide more effective and accurate capability in the near wall prediction. For the w velocity profiles (Fig. 2b), all the predictions show good agreement with the experiment.

The visualization of the flow features in the inclined ribbed duct and the turbulent coherent structures resolved by the DES is attempted by the comparison of the instantaneous coherent vorticity contours for several DES methods and steady RANS simulation as shown in Fig. 3. It is observed that only the large vortex behind the rib is captured in the steady SST simulation (Fig. 3a). However, much finer turbulent eddies have been resolved in DES simulations. In the DDES-SST solution (Fig. 3d), the massive flow separation and large shear layer over the rib and the helical vortex propagation towards the outer wall are clearly evident. Also, the breakup of the large coherent vortical structures into smaller eddies, and the chaotic turbulent eddies caused by the secondary flow impingement on the outer wall can be seen. The successful prediction of such resolved fine turbulent eddies provides the good evidence of the desired LES mode functionality in DES model for the present case of the separated vortical structures as well as the secondary flows. Compared to DDES-SST model, DDES-SA and DDES-RKE models didn't well capture the fine turbulence eddies in the secondary flows and its impinging area near the outer wall (Figs. $3 b \& 3 c$ ). It is attributed to the weakness of these two RANS models in the wall bounded complex turbulent flows.

An overview of the vortical flows appearing in the channel flow caused by the inclined rib is presented by the time averaged velocity contours over and downstream of the $5^{\text {th }}$ rib at the planes normal to the ribs in Fig. 4 . When the fluid approaches the rib, the flow separates from the bottom wall to avoid the obstacle, the rib. As a result, the streamlines of the main flow strongly curve upward. This curved flow generates a small recirculation vortex near the upstream bottom corner of the rib. All three steady and unsteady solutions of SA and SST based method captured this small vortex at the upstream corner, whereas only the DDESRKE does not show it. The narrowing of the cross-sectional area due to the rib 
forces the fluid velocity to increase. The maximum velocity is detected in the shear layer just downstream over the rib. A small flattened recirculation bubble on the top surface of the rib is caused by a boundary layer separation at the upstream rib top sharp corner. Then, just downstream over the rib a larger flow separation is developed from the rib downstream top corner because of the sharp widening. An elongated recirculation bubble takes place behind the rib, with an associated smaller counter-rotating vortex at the rib downstream bottom corner. Again only the DDES-RKE failed to produce all the small vortices near the wall corners. The shortcoming on the near wall turbulence prediction of the k- $\varepsilon$ based turbulence model is therefore witnessed. Further downstream the separated and narrowed flow re-expands in the duct and reattaches on the bottom wall. These flow features are in good agreement with the other observations [4-6].

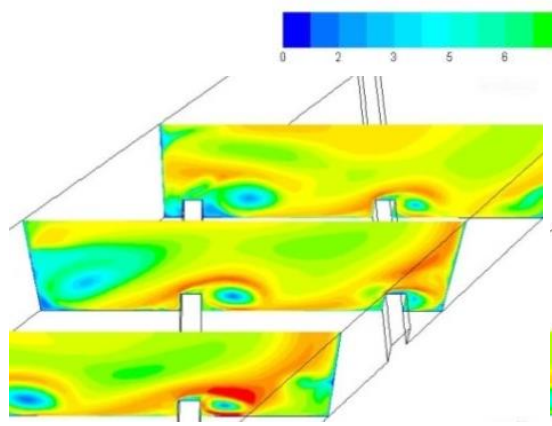

(a) Steady - SST

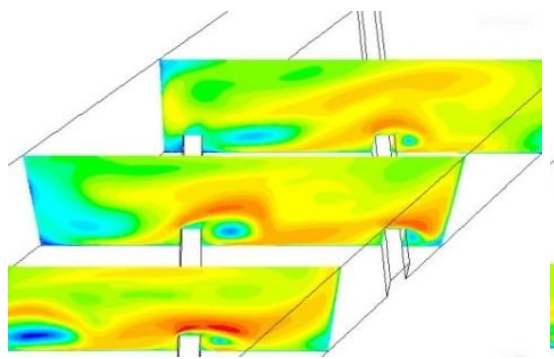

(c) DDES - RKE

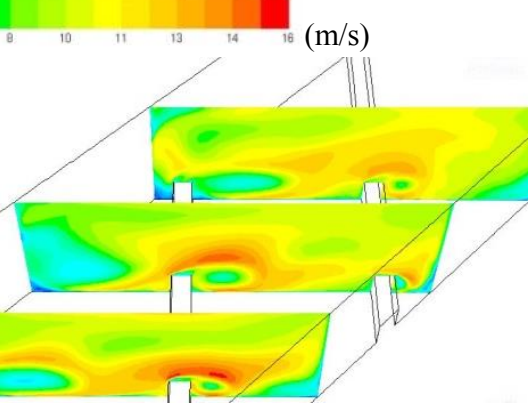

(b) DDES - SA

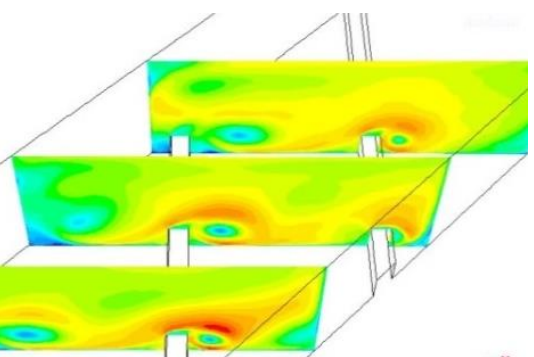

(d) DDES - SST

Figure 4. Time averaged velocity contours at the planes normal to the rib

As can be seen from the plots in Fig. 4, all simulations exhibit the similar overall flow features, such as large vortical flow behind the rib. However, the DDESSA and DDES-RKE models show thicker and over-accelerated shear layer over the rib. The steady SST model generally provides similar encouraging good results. However, thinner shear layer over the rib and the smaller size of the recirculation region is predicted. For the distance of the shear layer reattachment on the 
bottom wall, DDES-SST predicts a longer distance than those of DDES-SA and DDES-RKE. It is attributed to the shortcoming of these two models to resolve the smaller vortex at the corner. The result of steady SST simulation has the shortest distance of the reattachment because of the under estimation of the size of the vortical structure. By comparing the numerical simulations with these key flow features, the quality of the models has been analyzed. DDES-SST computation reasonably shows all the key flow features. However, DDES-RKE miss-represents all the small vortices previously described.

For all these unsteady DES simulations, the same mesh and the same initial condition, which is the converged solution of the steady SST simulation, are used. However, the results are quite depending on the RANS model involved in the DES method. In this kind of flow fields, a massive separating turbulent flow, generated by the geometrical factor, is dominant. Therefore, the modeling of the wall induced turbulent flow is of importance and an appropriate RANS model, which is suitable for the given flow features, should be selected in the DES method.

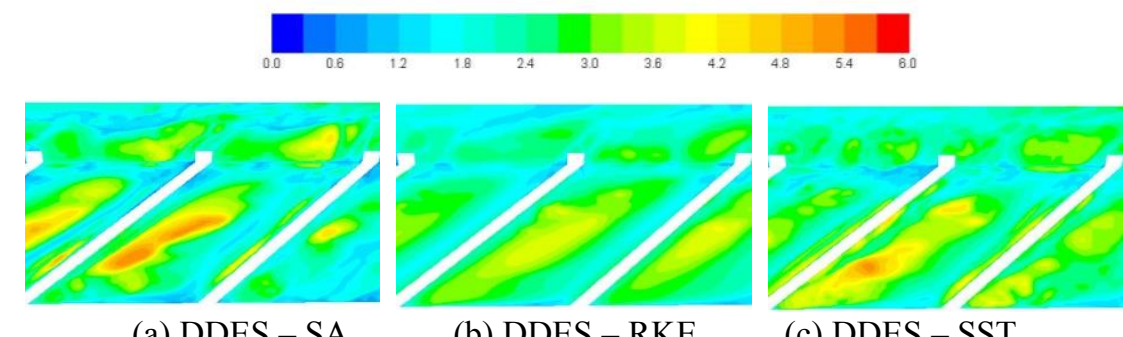

(a) DDES - SA

(b) DDES - RKE

(c) DDES - SST

Figure 5. Instantaneous Nusselt number distribution $\left(\mathrm{Nu} / \mathrm{Nu}_{0}\right)$ on the ribbed and the outer walls

\section{$\underline{\text { Heat transfer characteristics }}$}

The heat transfer results are presented in terms of an enhancement factor, $\mathrm{Nu} / \mathrm{Nu}_{0}$, compared to the heat transfer in a plane duct. Here, $\mathrm{Nu}$ is the Nusselt number at the wall, $\mathrm{Nu}=\mathrm{q}_{\mathrm{w}} \mathrm{D}_{\mathrm{h}} /\left[k\left(\mathrm{~T}_{\mathrm{w}}-\mathrm{T}_{\text {ref }}\right)\right]$, where $\mathrm{q}_{\mathrm{w}}$ is the given wall heat flux of the heating wall, and $k$, is the thermal conductivity. $\mathrm{Nu}_{0}$ is obtained from the Dittus-Boelter correlation for a plane duct under the heating boundary condition: $\mathrm{Nu}_{0}$ $=0.023 \mathrm{Re}^{0.8} \mathrm{Pr}^{0.4}$. Instantaneous Nusselt number distributions on the bottom ribbed wall and the outer side wall of three DES solutions are shown in Fig. 5. On the bottom wall given in Fig. 5, the numerical results show that the high heat transfer regions coincide with the region of secondary helical vortical flow impingement rather than the region of the reattachment of the shear layer. The high heat transfer region is widen towards the outer side wall and moves downstream as the secondary vortical flow is enlarged and move downstream towards the outer wall. These vortices enhance turbulent mixing and increase heat transfer coefficient. As shown in Fig. 3, these large coherent vortical structures are not well cap- 
tured in DDES-SA and DDES-RKE cases. Consequently, the DDES-RKE underpredicts the heat transfer coefficient. Also, the maximum heat transfer regions of DDES-SA and DDES-RKE are rather in the middle region between two side walls, contrary to the maximum heat transfer region near the inner wall of DDESSST solution. It is highly correlated with the stronger vortex behind the rib in upstream region (near the inner wall) as shown in Fig. 3. Eventually, it is evident that the accuracies of turbulent flow structures prediction and the heat transfer performance prediction are closely related.

The averaged heat transfer coefficients on the ribbed wall are summarized in Table 1. The calculated results are compared with the experimental measurement [4]. Note that the uncertainty of the experimental measurement on the heat transfer coefficient was given as $\pm 8 \%$. In this context, generally, all the numerical predictions show the agreement within the acceptable range of $\pm 12 \%$. As discussed in the former section, both of DDES-SA and DDES-RKE under-predict by $11 \%$ and $12 \%$, respectively. As expected, DDES-SST shows the best agreement of $4 \%$ discrepancy. The steady SST solution over-predicts the heat transfer coefficient.

The detailed distribution of the time averaged heat transfer coefficients of DDES-SA, DDES-RKE, and DDES-SST on the bottom ribbed wall and the outer side wall are compared with the results of the steady SST model, the LES result [7] and the experiment [4] in Fig. 6. From the heat transfer patterns on the ribbed surfaces in Fig. 6, it can be seen that DDES-SST shows better capability of heat transfer prediction than others in the present case, in terms of the maximum value of Nusselt number and its location. The heat transfer is high downstream of the rib, as a result of the strong vortical flow. As the vortical flow moves along the rib from the inner side wall towards the outer side wall, the vortex core increases in size, as well as moves downstream from the rib and upward from the bottom wall, simultaneously (as shown in Figs 3d and 4d). However, the strength of the vortex decreases as it moves from inner to outer side walls. Therefore, the higher heat transfer occurs in the downstream vicinity of the rib near the inner side wall and then the Nusselt number decreases along the downstream towards the outer wall.

The location of the maximum Nusselt number and its value are DES model dependant. DDES-SST shows similar Nusselt number results to the LES result and the experiment. However, generally the DES models under-predict Nusselt number. The locations of the maximum $\mathrm{Nu}$ in DDES-SA and DDES-RKE cases are further downstream from the inner wall and the rib than those of the DDES-SST and the experiment.

Table 1. The averaged heat transfer results on the bottom ribbed wall

\begin{tabular}{l|cc}
\hline & $N u / N u_{0}$ & Discrepancy \\
\hline Experiment [9] & 2.78 & - \\
Steady SST & 2.918 & $5 \%$ \\
DDES-SA & 2.468 & $-11 \%$ \\
DDES-RKE & 2.436 & $-12 \%$ \\
DDES-SST & 2.659 & $-4 \%$ \\
\hline
\end{tabular}




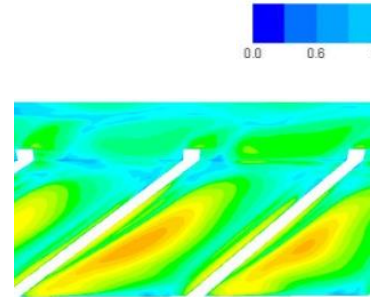

(a) Steady - SST

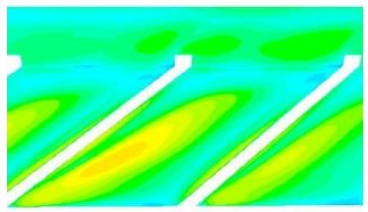

(d) DDES - SST

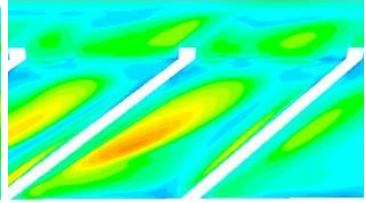

(b) DDES - SA

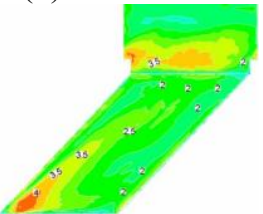

(e) LES result in [7]

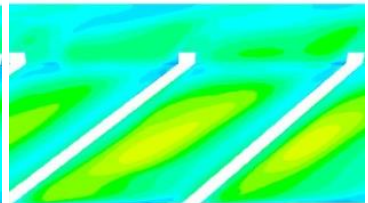

(c) DDES - RKE

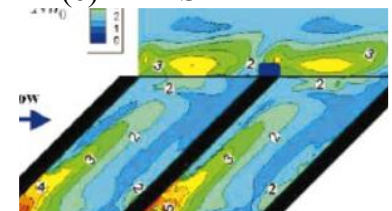

(f) Experiment in [4]

Figure 6. Time averaged Nusselt number distribution $\left(\mathrm{Nu} / \mathrm{Nu}_{0}\right)$ on the ribbed and the outer walls

The secondary flows play a major role in the heat transfer on the side walls, as it can be seen in Figs. 5 and 6 . The heat transfer on the outer side wall is enhanced by two vortical flows; the first by the accelerated shear layer right above the rib, the second by the impingement of the helical vortical flow between the ribs. DDES-SST shows the closest $\mathrm{Nu}$ distribution on the outer wall to the experiment. DDES-RKE under-predicts the Nu values. Also, the higher heat transfer enhancement region on the outer side wall of these models occurs further downstream than those of DDES-SST and the experiment.

\section{Conclusion}

Several DES approaches with different RANS turbulence models (DDES-SA, DDES-RKE, DDES-SST) have been scrutinized for the predictions of turbulent large vortical flow and heat transfer in the $45^{\circ}$ angled ribbed channel at a Reynolds number of 50,000. The significant effect of RANS model on the accuracy of the DES prediction has been shown. The results of DDES-SST were consistently found to be the best compared to the others in the present test case.

The helical vortex and the secondary flow caused by the inclined rib have a major role in the heat transfer enhancement. The maximum heat transfer on the ribbed wall occurs at the origin of the vortex just downstream of the rib near the inner side wall. On the outer side wall, the region of the helical vortex impingement has higher Nusselt number.

It is concluded that the selection of RANS modeling for the wall induced turbulent flow is of importance in the performance of DES method. DDES-SST can be used with reasonable accuracy to predict the flow and heat transfer in complex internal flow configurations. Even though DDES-SST shows good prediction in the turbulent flow structures, it still has somewhat discrepancy in heat transfer predic- 
tion. Therefore, further investigation is required to improve the capability of DES method, particularly in terms of heat transfer prediction.

\section{REFERENCES}

[1] Sewall, E. A., Tafti, D. K., Graham, A. B. and Thole, K. A., 2006, "Experimental validation of large eddy simulations of flow and heat transfer in a stationary ribbed duct," International Journal of Heat and Fluid Flow, 27(2), p. 243-258.

[2] Fronhlich, J. and von Terzi, D., 2008, "Hybrid LES/RANS methods for the simulation of turbulent flows," Progress in Aerospace Sciences, 44(50), p. 349-377.

[3] Viswanathan, A. K. and Tafti, D. K., 2007, "Investigation of detached eddy simulations in capturing the effects of Coriolis forces and centrifugal buoyancy in ribbed ducts," Journal of Heat Transfer, 129(7), p. 778-789.

[4] Chanteloup, D., Juaneda, Y., and Bolcs, A., 2002, "Combined 3-D flow and heat transfer measurements in a 2-pass internal coolant passage of gas turbine airfoils," Journal of Turbomachinery, 124(4), p. 710-718.

[5] Bonhoff, B., Parneix, S., Leusch, J., Johnson, B. V., Schabacker, J. and Bolcs, A., 1999, "Experimental and numerical study of developed flow and heat transfer in coolant channels with 45 degree ribs," International Journal of Heat and Fluid Flow, 20(3), p. 311-319.

[6] Labbe, O., 2013, "Large eddy simulation of flow and heat transfer in a ribbed duct," Computers \& Fluids, 76, p. 23-32.

[7] Abdel-Wahab, S. and Tafti, D. K., 2004, "Large eddy simulation of flow and heat transfer in a staggered $45^{\circ}$ ribbed duct," ASME Turbo Expo 2004, GT2004-53800. 